\title{
Game Theoretical Analysis of Collaborative Social Applications
}

\author{
Ahmed Al-Dhanhani*, Rabeb Mizouni ${ }^{\dagger}$, Hadi Otrok ${ }^{\dagger}$, and Ahmad Al-Rubai* \\ ${ }^{*}$ Etisalat BT Innovation Centre, P.O. Box 127788, Abu Dhabi, UAE \\ Email: ahmed.aldhanhani@kustar.ac.ae \\ Email:ahmad.al-rubaie@bt.com \\ ${ }^{\dagger}$ Khalifa University of Science, Technology and Research, P.O. Box 127788, Abu Dhabi, UAE \\ Email: rabeb.mizouni@kustar.ac.ae \\ Email: hadi.otrok@kustar.ac.ae
}

\begin{abstract}
During the last decade, social applications have witnessed a rapid growth in their use. Millions of people are utilising them on a daily basis in order to share their experience, information and to communicate with their family members and friends. Lately, these technologies have been used to foster collaboration in education, however, it is a case of hit and miss and without established techniques to ensure or replicate success. A number of factors contribute to the limited success of such groups, one such factor is the presence of selfish members. A selfish user adopts a free riding behaviour that takes advantage of the collaborative group without contributing back. Such a behaviour will affect the group's sustainability and affect the participants willingness to contribute. To improve the survival of educational groups in social applications, free riding behaviour needs to be studied, its impact on the group survivability assessed and then addressed. In this paper, we formally analyse the impact of the free riding behaviour by means of repeated game theory where classical and generous Tit-for-Tat are used.
\end{abstract}

Index Terms-Game Theory, Social Applications, Tit-for-Tat, Free Riding, Collaborative Learning, Collaborative Groups.

\section{INTRODUCTION}

Recently, social applications have been gaining more popularity on the Internet and a great deal of user generated content has been produced with a plethora of users consuming them. Many have seen the opportunities of using the popularity of social applications in different fields such as education; and educators have already started working on using social applications to foster collaboration among groups of learners. These groups can exist in forums, emails, chat systems, questions and answers sites, and most recently social networking sites.

Till now, the success of social applications for collaboration has not been consistent and the ability of these social applications to stimulate their subscribers to contribute varies greatly. The work on encouraging the participants to contribute has been more of an art than science [1]. There is a combination of factors that have been studied and claimed to result on guaranteeing the active contribution of the participants ranging from psychological to economical views [1]. It is not an easy task to create a controlled study to test one factor in separation from the others in a real online community. In this paper, we adopt the economical view by assuming that users are rational agents aiming at maximising their benefits. Their benefits increase when other users collaborate with them.
Collaboration is, in fact, the success key for educational social applications. Unfortunately, over time learners' participation may diminish or even stop because they may lose interest on the theme of the discussion and/or they do not get satisfactory answers to their requests. Selfish behaviour could also arise where some participants will try to take advantage of the group and request help from the other participants while ignoring answering requests of others to maximise their benefit. Such situation is referred to as the tragedy of the commons [2]. In the long run, the existence of such free riding behaviour may demotivate participants from collaborating and may affect the survivability of the group.

To analyse such a free riding behaviour and its impact on group collaboration, we use game theory to formulate the interaction among all participants as a repeated non-cooperative game based on Tit-for-Tat strategy. In our investigation, we create a model where a number of participants formed a group inside a social application for the purpose of collaborating. We simulate the results of the participants adapting different behaviours. Using classical and generous Tit-for-Tat strategies, we show the impact of having free riding behaviour on social applications' groups. In summary, the contributions of our paper are:

- Modelling a collaborative group in a social network with repeated game theory

- Simulating the participants behaviours using different Tit for Tat strategies, and

- Analysing the impact of free riding in collaborative groups.

The rest of the paper is structured as follows. Section II presents the game theory model. In section III, we show the different Tit for Tat strategies. Section IV presents some related work in the field of game theory and social applications. Finally section $\mathrm{V}$ concludes the paper.

\section{Social Network Group: Game Definition}

In collaborative social applications, users cooperation can take many forms such as answering others' requests, participating in discussions or simply commenting on others' contributions. However, participants can also choose to defect from cooperating and contributing. 
Using game theory, this situation of cooperating and defecting can be modelled as a non-zero non-cooperative game [3]. It is modelled as a non-zero because the benefit here is collaborating and sharing knowledge and this kind of benefit can not be transferred but rather shared between two persons. Also, it is modelled as a non-cooperative because each person makes his decision independently from the others.

TABLE I

PRISONER'S DILEMMA PAYOFF MATRIX

\begin{tabular}{|c|c|r|}
\hline & Cooperate & Defect \\
\hline Cooperate & $\mathrm{R}(-1), \mathrm{R}(-1)$ & $\mathrm{S}(-10), \mathrm{T}(0)$ \\
\hline Defect & $\mathrm{T}(0), \mathrm{S}(-10)$ & $\mathrm{P}(-5), \mathrm{P}(-5)$ \\
\hline
\end{tabular}

Such games are usually modelled against the famous Prisoner's Dilemma game invented by two Rand corporation scientists in 1950s [4]. The prisoner's dilemma game represents the situation of two criminals caught by the police at the same time. These criminals have two strategies to independently select from. They can either confess (defect) or not (cooperate). The results of the possible outcomes is outlasted in table I where:

1) R: (Reward) is to be sentenced for 1 year

2) P: (Punishment) is to be sentenced for 5 years

3) S: (Sucker) is to be sentenced for 10 years

4) $\mathrm{T}$ : (Temptation) is to be set free.

These constants must satisfy the following two conditions:

1) $T>R>P>S$

2) $2 R>T+S$.

In the prisoner's dilemma game, the dominant strategy technique is used to promote the best strategy to be used by a player regardless of the other player decision. By using it, we can conclude that the best strategy for a criminal when both criminals do not know the other's decision is to "defect" to avoid the sucker which is the worst case since $T>R>P>S$. However, in the case of playing this game for infinite number of times, then the best strategy will be changed to "cooperate" since $2 R>T+S$.

The prisoner's dilemma game as is cannot resemble a collaborative group where decisions between two participants are not simultaneous but taken in two different stages. A modified version of the prisoner's dilemma game which is the iterated asynchronous prisoner's dilemma game has been introduced in the literature. To accommodate these changes in the game, we can use the model proposed in [5] summarised in table II.

These values should be calculated so that they do not violate the previously mentioned two conditions of the prisoner's dilemma.

\section{A. Collaborative Game Settings}

In this section we explain the settings and introduce our assumptions that we considered when formulating the game. Consider that we have a group of $(N)$ participants in a social
TABLE II

ASYNCHRONOUS GAME MODEL

\begin{tabular}{ll}
\hline Payoffs & \\
\hline Reward & $R=a+b$ \\
Punishment & $P=c+d$ \\
Temptation & $T=c+b$ \\
Sucker & $S=a+d$ \\
\hline Considering & \\
\hline$a$ & The cost of cooperating in a turn \\
$b$ & The reward from being cooperated with in \\
& a turn \\
$c$ & The cost of defecting in a turn \\
$d$ & The cost of not being cooperated with \\
\hline
\end{tabular}

application, each user is a member of $m$ groups and posts made by users are restricted to groups. Each user is able to:

1) Post a request.

2) Answer others' requests.

3) Ignore a request because s/he is not able to answer it.

4) Ignore a request although $\mathrm{s} / \mathrm{he}$ is able to answer it. Such a behaviour is known as "selfish" or "free riding".

We also assume that :

1) The social application is keeping track of all requests and responses of each participant.

2) The social application is updating each user with the cooperation status of the other users.

3) The group lifetime is infinite.

4) All participants are active.

Our game will run for 10000 iterations. At each iteration $(t)$, $\frac{1}{10}$ of the users in the group will be chosen randomly to have requests $Q_{i q}(t)$. The rest of the users can either respond to such requests or ignore them. This can happen because users can be selfish or because may not be to answer. Our model considers ignoring requests as a defection regardless of the reason.

In total, we have $(N)$ participants where $\left(N_{c}\right)$ of them are cooperative and $\left(N_{s}\right)$ are selfish where $N=N_{c}+N_{s}$. For each interaction between two participants, a non-requesting cooperative participant $j$ would offer one useful response with a probability of $P_{a}: 0<P_{a}<\frac{1}{10}$. Each participant has his own $P_{a}$ value ranging from 0 to $\frac{1}{10}$. The values of $P_{a}$ are distributed normally between the participants to simulate the difference between them. The variability of $P_{a}$ itself simulates the probability of a participant ability to answer a request because he does not have enough knowledge, he does not have time to answer it or he did not have the chance to see the request.

A summary of these game elements is described in Table III.

In the following, we define our game parameters that can fulfil the conditions of the asynchronous repeated prisoners dilemma game stated in table II:

1) Gain from getting a response, $b=10$ 
TABLE III

SUMMARY OF THE GAME ELEMENTS

\begin{tabular}{ll}
\hline Game Model Elements & $\begin{array}{l}\text { Participants of a group. They can be } \\
\text { selfish or cooperative. }\end{array}$ \\
Cooperate & $\begin{array}{l}\text { Responding to other participants re- } \\
\text { quests/posts } \\
\text { Ignoring/not answering other partici- } \\
\text { pants requests }\end{array}$ \\
Selfish User & $\begin{array}{l}\text { Posts new questions to others but do not } \\
\text { answer others' requests }\end{array}$ \\
$N_{s}$ & $\begin{array}{l}\text { Number of selfish users } \\
\text { Requests from others and answer others' } \\
\text { requests }\end{array}$ \\
$N_{c}$ & $\begin{array}{l}\text { Number of cooperative users } \\
P_{q}\end{array}$ \\
$\begin{array}{l}\text { Number of requests at an iteration } \frac{1}{10} \\
\text { of the total number of users }\end{array}$ \\
$\begin{array}{l}\text { The probability of a player being able } \\
\text { to respond to requests and ranges from } \\
0 \text { to } \frac{1}{10}\end{array}$ \\
Number of iterations $i$
\end{tabular}

2) Cost of responding a request, $a=-1$

3) Loss from not getting any response to a request, $d=-10$

4) Cost of ignoring a request, $c=0$

Note that the parameter $a$ is given a negative value to represent the cost of responding to a request since it requires effort and time to write an answer. According to our settings, $c$ is greater than $a$. It reflects the fact that ignoring a request gets a higher benefit at one time. Furthermore, $c-a$ is less than $b-d$ showing that the cost of responding is less than the benefit of getting a response. Therefore, for the longer term, rational users are better off cooperating with each other. These parameters ensure that the results would give an indication on the survivability and the efficiency of the group for all cooperative users. Also, it is worth to note that these parameters show the calculations made per request.

\section{B. The impact of Selfish behaviour}

To show the impact of having selfish users, let us consider two scenarios :

1) A scenario where there are 100 participants and all of them are cooperative participants

2) A scenario where there are 100 participants, $80 \%$ of them are cooperative and the rest are selfish

The comparison between these two scenarios should show us the effect of having selfish users on the total gain of cooperative users.

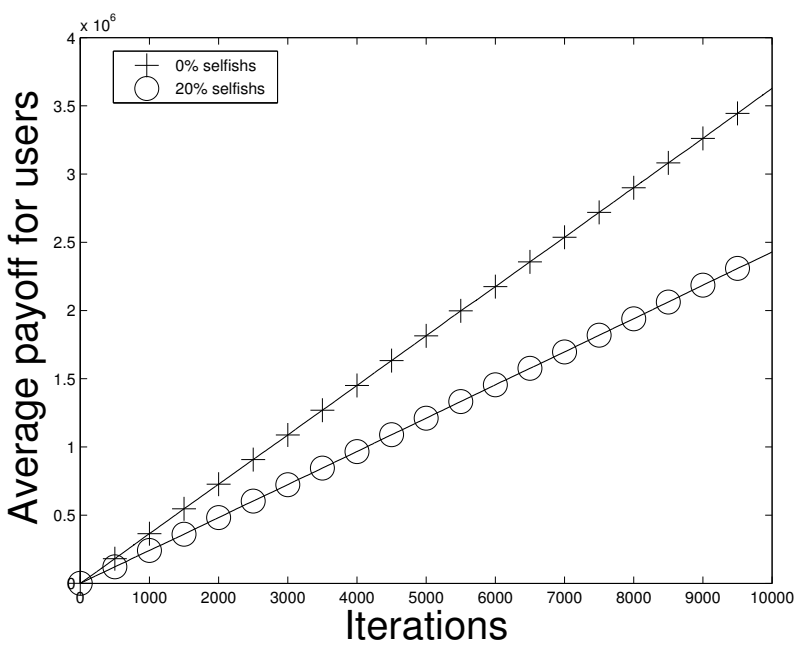

Fig. 1. Upper bound of total cooperative gain with 100 users of $0 \%$ and $20 \%$ selfish participants

By comparing the gain of $80 \%$ of the cooperative participants from the first scenario with $80 \%$ cooperative participants of the second scenario, we got the results shown in figure 1.

The gap between the curves of the two scenarios is the loss to the cooperative users' gain inflicted by the existence of selfish users in the group. This loss can be turned into gain if the selfish users were somehow forced to cooperate.

\section{THE TIT FOR TAT STRATEGIES}

In this section and through the use of simulations we aim to analyse the impact inflected by selfish users to the gain of cooperative users and how increasing the percentages of selfish users can affect the system and to what extent. A summary of those scenarios is in Table IV.

\section{A. Model I: Traditional Tit-for-Tat with fixed history}

The first Tit-for-Tat model we are investigating is the traditional one. In this model the player should look back at the previous move by the other player and act upon it. In an iterated game, we will assume that the player can look at $k$ number of moves of the other player. Therefore, we assume that we have a player $j$ who keeps the historic record $H_{j i}$ with another player $i$ for a specific number of movements $k$. Each $H_{j i}(t)$ value corresponds to the decision $D_{i j}(t)$ taken by player $i$ to cooperate or not with player $j$ at time $t$. Then depending on the historic record kept from $H_{j i}(1)$ to $H_{j i}(k)$ player $j$ will make a decision $D_{j i}(t)$ to cooperate with $i$ or not. If the collective value from $H_{j i}(1)$ to $H_{j i}(k)$ is bigger than $\frac{k}{2}$, player $i$ will be considered cooperative and player $j$ will try to cooperate with player $i$; otherwise, player $j$ will defect. Of course, this also depends on player $j$ having the answer 
TABLE IV

SCENARIOS

\begin{tabular}{ll}
\hline Strategy & \\
\hline Classical TFT & $\begin{array}{l}\text { A cooperative participant will cooperate with another partic- } \\
\text { ipant only when the history of cooperating between the two } \\
\text { is more than the history of defection. }\end{array}$ \\
Generous TFT & $\begin{array}{l}\text { A cooperative participant will cooperate with another partic- } \\
\text { ipant conditionally when he finds that he cooperated at least } \\
\text { once for a specific period and unconditionally in a regular } \\
\text { basis to avoid deadlocks }\end{array}$
\end{tabular}

$A_{j, i}(t)$ to the request of player $i$. This can be resembled by equation 1 .

$$
D_{j, i}(t)=\min \left\{A_{j, i}(t), \max _{1 \leqslant h \leqslant k} H_{j, i}(h)\right\}
$$

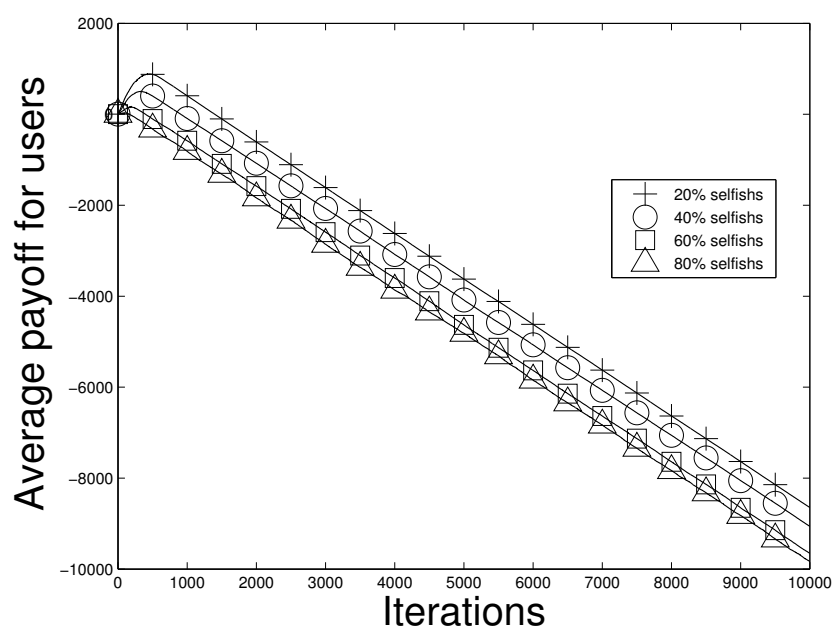

Fig. 2. Comparison between the different percentages of selfish users in classical tit for tat

Fig. 2 shows the average cooperative users gain in the case of using the classical tit for tat strategy with 100 users, with the lines representing $20 \%, 40 \%, 60 \%$ and $80 \%$ of selfish users. It can be seen that in all the plots, the total payoff of the cooperative users reached a deadlock and started decreasing. This is due to the fact that $1-\operatorname{Average}\left(P_{a}\right)$ of the users even if they were cooperative, are not able to respond to the request. Consequently, a cooperative user will start building a history of bad relationship with another cooperative user which leads to both of them ending up with the same history of bad relationship causing a mutual deadlock. Due to the payoff of -10 for a user not getting any answer, the total payoff keeps on decreasing at the end. We can see that the more selfish users in a group, the faster the payoff reaches a deadlock.

Conclusion: Modelling collaboration in a group of learners with a fixed history tit-for-tat strategy leads to decreased collaboration even among cooperative learners.

\section{B. Model II: The Generous Tit-for-Tat}

Since the classical Tit for Tat ends up with a deadlock, another enhanced Tit for Tat strategy that is able to avoid the deadlock was presented in the literature which is the generous Tit for Tat. It is based on a relaxed criterion for cooperation. A cooperative player $j$ will cooperate with player $i$ at a regular basis of $k$ movements regardless of their previous history from $H_{j i}(1)$ to $H_{j i}(k)$. Moreover, only one cooperation(one answer) in the past $k$ decisions is enough to consider the other player cooperative, instead of $\frac{k}{2}$ cooperations in the previous classical tit for tat model. This corresponds to the equation 2.

$$
D_{j, i}(t)=\left\{\begin{array}{c}
A_{j, i}(t)-i f H_{j i}(h) \neq \varnothing, \text { for some } \\
A_{j, i}(t)-\text { every } k \text { moves }
\end{array}\right.
$$

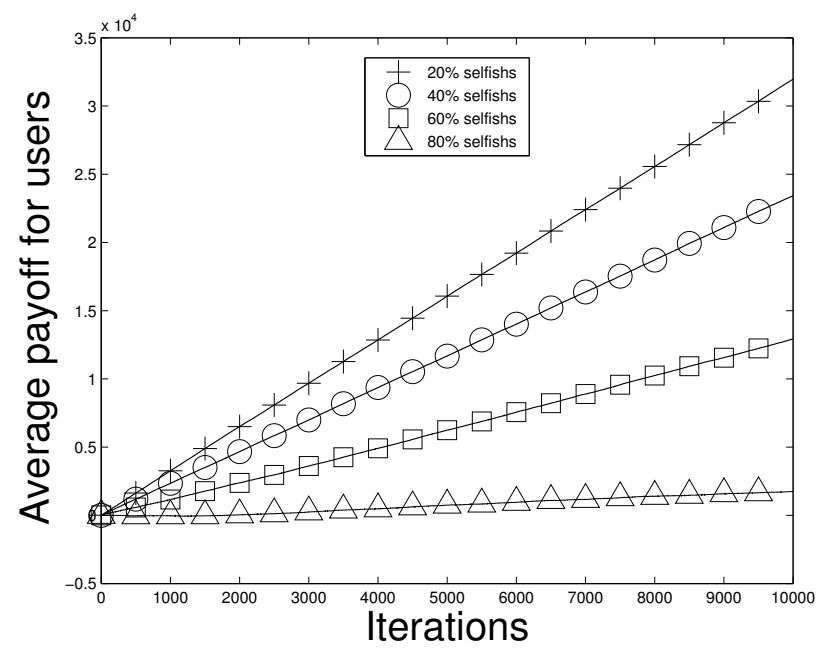

Fig. 3. Comparison between the different percentages of selfish users in generous tit for tat

Fig. 3 shows the cooperative users gain in the case of using the generous tit for tat strategy with 100 users, with the lines representing $20 \%, 40 \%, 60 \%$ and $80 \%$ of selfish users. As we can see, this strategy does not lead to a deadlock as observed in the previous one. This is due to the generous behaviour preventing the cooperative users from reaching a mutual deadlock. In fig. 4, we see a comparison between 


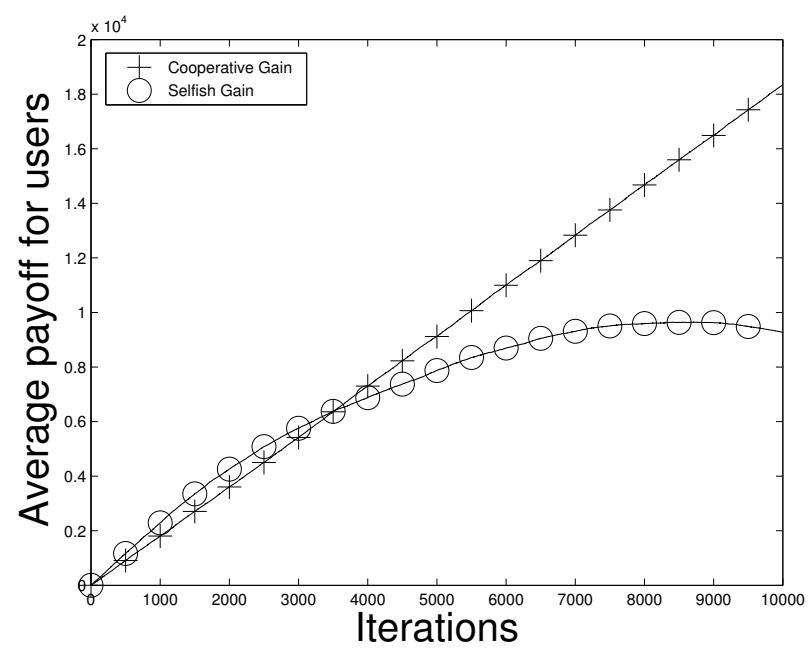

Fig. 4. Comparison between the selfish and cooperative gain in generous tit for tat with $50 \%$ selfish users

the gain of cooperative users and selfish users in the case of having 1 offer and 50\% selfish users. This result shows that the strategy is good in the case of having selfish users that do not cooperate at all. Although the generous behaviour results in them getting an answer every $k$ turns, their cumulative loss of not getting any answer is much higher which results in the drop of their total gain.

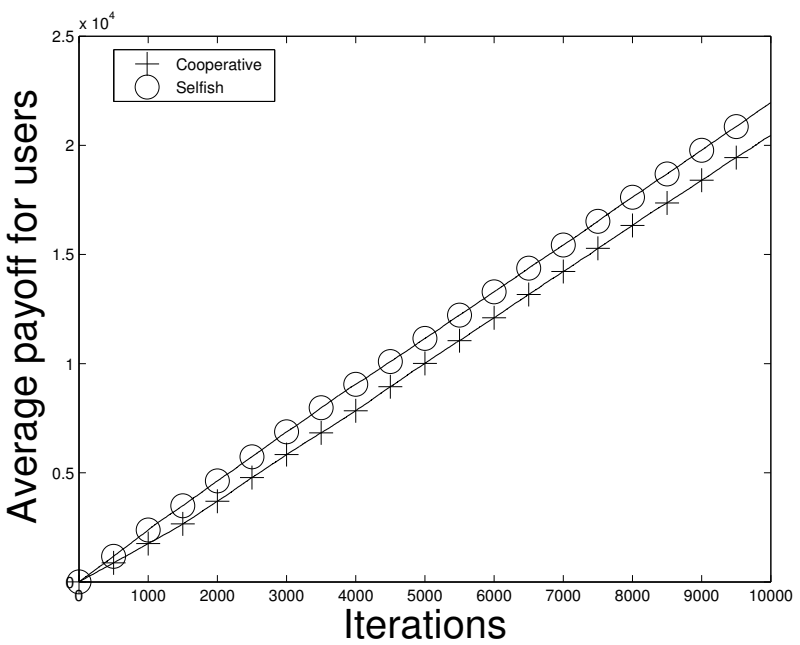

Fig. 5. Comparison between the mutated selfish and cooperative gain in generous tit for tat with $50 \%$ mutated selfish users

However, selfish users may mutate their behaviour and cooperate once every time their history of movements with another user is full of defection to imitate the cooperative user behaviour. The result of such behaviour is shown in fig. 5. It demonstrated that even with partial cooperation, the number of times selfish users cooperated did not go above $\frac{1}{5}$ of the number of times the cooperative users did, the gain of selfish users is slightly higher than the cooperative users since they keep their contributions to a minimum.

One way to force selfish users to cooperate more is by

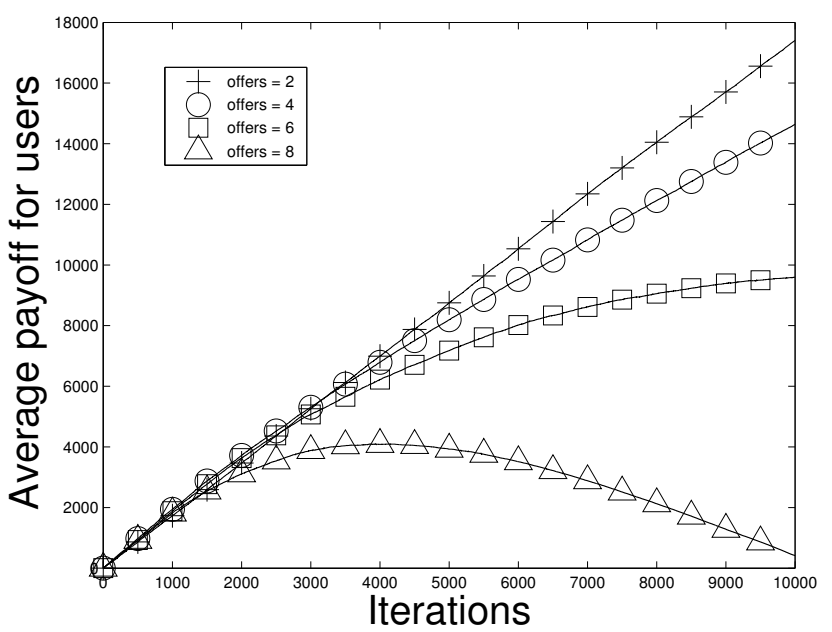

Fig. 6. Comparison between the different offers in generous tit for tat with $50 \%$ selfish users

increasing the number of required cooperations for a user to be considered cooperative. However, this kind of conditions will affect the gain of cooperative users significantly as apparent in fig. 6. It is due to the fact that more cooperation is required from all the users regardless of their ability of answering the questions.

To address this problem, we suggest to increase the maximum

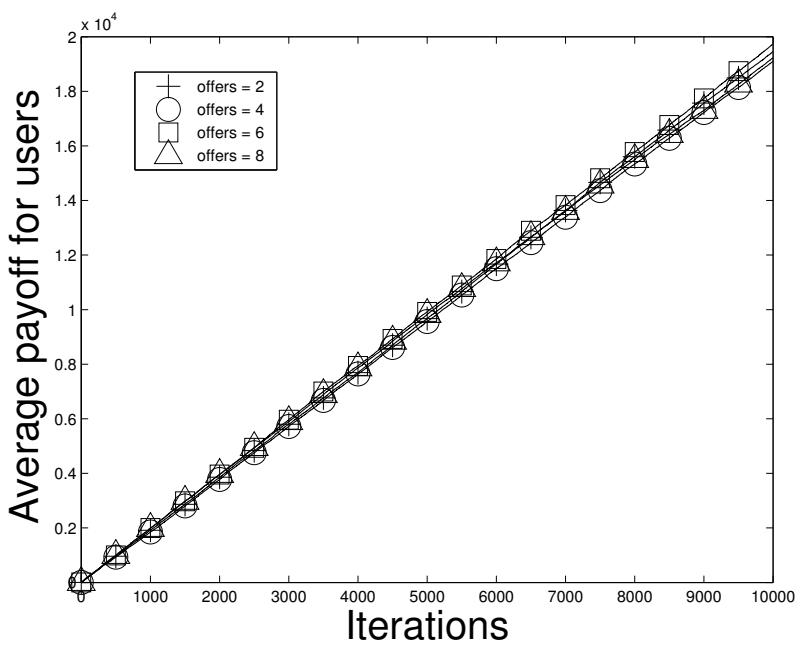

Fig. 7. Comparison between the different offers in generous tit for tat with $50 \%$ selfish users with $k=150$ movements

number of movements $k$ in the history since the affect of increasing the requested number of cooperations or answers decreases with the increasing of $k$. The result of such an 
example, comparing 1 cooperation and 2 cooperations with 100 movements in the history is shown in fig.7. Although the strategy became more beneficial with the increase of the history records; however, large history records have a technical disadvantage if they were to be implemented and are better be avoided in such systems.

Conclusion: Modelling collaboration in a group of learners with a generous tit-for-tat strategy increases the overall collaboration. however, selfish users are able to take advantage of the strategy easily. This will result on demotivating the users from becoming cooperative.

\section{Discussion}

In the Tit for Tat strategy, the player makes his decision based on the other player's previous moves. However, as proven by our simulation, a strategy such as previously described will not always solve the problem of the free riding behaviour that groups are suffering from. In a collaborative group, there are situations where a participant will have a request and the other cooperative participants, while they want to help, they simply do not have the knowledge/expertise to do so. This stochastic behaviour could lead to a deadlock of defection between participants as seen in Fig. 8 which shows the average payoff over time for the studied strategies. Because of the deadlock group may reach, the classical Tit for Tat strategy is not able to survive even with only $20 \%$ selfish users.

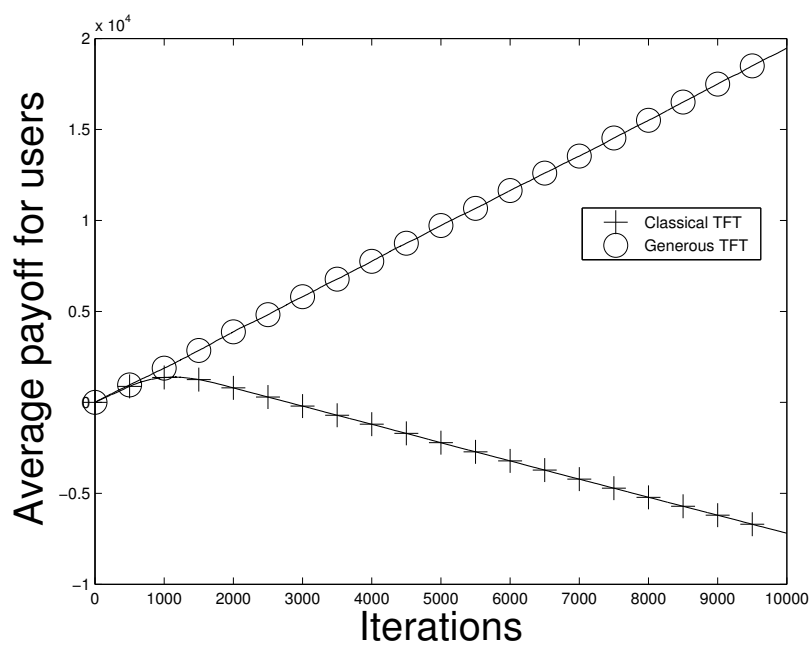

Fig. 8. Comparison between the different Tit for Tat strategies in cooperative users gain with $50 \%$ selfish users

While the generous tit for tat contributes towards addressing these issues and prevents possible deadlock because the cooperative participants will be generous in a regular basis and give without expecting receiving any benefit, this strategy is not efficient in stopping selfish behaviour since it is easy to take advantage of. As we see in fig.9, the generous Tit for Tat even maximises the gain of selfish users compared to the other strategy.

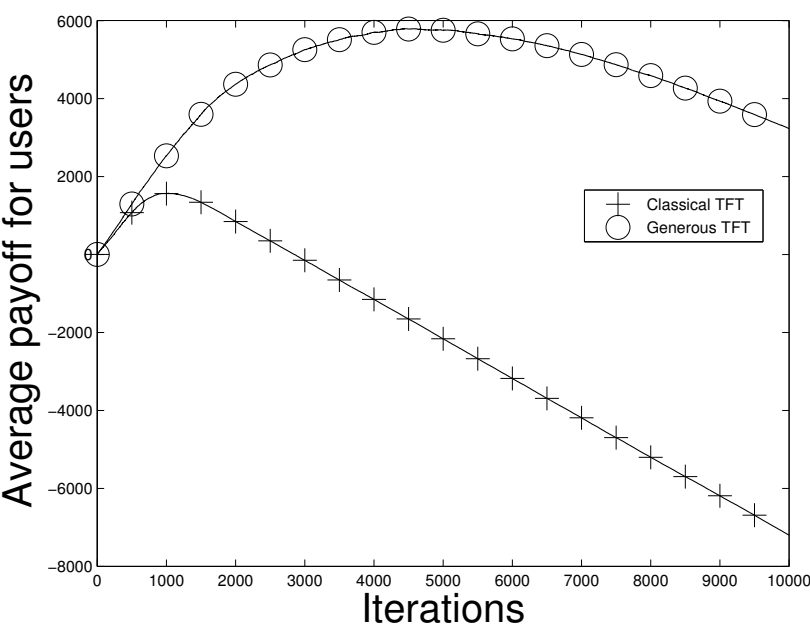

Fig. 9. Comparison between the different Tit for Tat strategies in selfish users gain with $50 \%$ selfish users

To foster the cooperation in collaborative groups, any collaborative social application should:

- maximise the gain of cooperative participants,

- minimise the gain of selfish users to prevent free riding behaviour, and

- motivate selfish users to cooperate in order to be of benefit to the group, rather than eliminating them from the group.

\section{RELATED WORK}

In line with our study, some studies analysed the problem of motivating users, selfish and cooperative, in the context of online communities in general [1]. In these studies, most of the attempts to analyse the problem are experimental with many uncontrolled variables in established online communities. There are few attempts in using computer simulations to predict the effects of different incentive mechanisms in online communities [6] [7]. Each focused on the results of applying specific incentive mechanism techniques according to economics, behavioural economics and psychology theories. [6] studies the user motivation and incentive mechanisms for participants of an online community for sharing articles and URLs. [7] simulates the effects of different kinds of moderation on the participation of the participants of an online community using an agent based model.

In our model, we use the approach of game theory and more specifically, the prisoner's dilemma game and tit-for-tat strategies, to simulate the behaviour of the participants and analyse it.

It is worth noting that game theory was used extensively to study the problem of selfish users in the fields of wireless ad-hoc networks, virtual private networks and peer-to-peer networks as well [8], [9], [10]. 


\section{CONCLUSION}

In this research we proposed to model the selfish behaviour in collaborative groups of social applications using game theory. By simulating the groups' interactions of posting requests and receiving answers, we explored the possibilities and the outcomes of the users using known Tit for Tat strategies of the game theory. Through the simulation of the Classical and the Generous Tit-for-Tat strategies, we have shown that they do not solve the free riding behaviour problem groups may suffer from. Our analysis raises the need for social applications to have features that motivate selfish users to cooperate under the threat of being punished by other cooperative users if they are not of benefit to the group.

\section{REFERENCES}

[1] J. Vassileva, "Motivating participation in social computing applications: a user modeling perspective," User Modeling and User-Adapted Interaction, pp. 1-25, 2012.

[2] G. Hardin, "The tragedy of the commons," New York, 1968.

[3] P. Sloep, "Fostering sociability in learning networks through ad-hoc transient communities," Computer-Mediated Social Networking, pp. 6275, 2009.

[4] R. Axelrod and W. Hamilton, "The evolution of cooperation," Science, vol. 211, no. 4489, p. 1390, 1981.

[5] M. NowAKf and K. SigmundJ, "The alternating prisoner's dilemma," $J$. theor. Biol, vol. 168, pp. 219-226, 1994.

[6] Y. Mao, J. Vassileva, and W. Grassmann, "A system dynamics approach to study virtual communities," in System Sciences, 2007. HICSS 2007. 40th Annual Hawaii International Conference on, pp. 178a-178a, IEEE, 2007.

[7] Y. Ren and R. Kraut, "A simulation for designing online community: Member motivation, contribution, and discussion moderation," Information Systems Research, 2011.

[8] V. Srivastava, J. Neel, A. MacKenzie, R. Menon, L. DaSilva, J. Hicks, J. Reed, and R. Gilles, "Using game theory to analyze wireless ad hoc networks," Communications Surveys \& Tutorials, IEEE, vol. 7, no. 4, pp. 46-56, 2005.

[9] J. Robert, H. Otrok, A. Quttoum, and R. Boukhris, "A distributed resource management model for virtual private networks: Tit-for-tat strategies," Computer Networks, 2011.

[10] D. Peng, W. Liu, C. Lin, Z. Chen, and X. Peng, "Enhancing tit-for-tat strategy to cope with free-riding in unreliable p2p networks," in Internet and Web Applications and Services, 2008. ICIW'08. Third International Conference on, pp. 336-341, IEEE, 2008. 